\title{
A GENERALIZATION OF THE NAGUMO UNIQUENESS CRITERION
}

\author{
THOMAS C. GARD
}

\begin{abstract}
The classical Nagumo criterion for uniqueness of solutions of the initial value problem $x^{\prime}=F(t, x), x\left(t_{0}\right)=x_{0}$, assumes continuity of $F$ as well as a modulus of continuity condition involving $1 / t$. Recently, it has been shown that $1 / t$ can be replaced by $\alpha / t$, for $\alpha>1$, or by $1 / t^{2}$ provided that the continuity condition on $F$ is suitably modified; in each case it is required that $F(t, x)$ at least approach a limit at a certain minimal rate as $(t, x)$ tends to the initial value. In this note we extend both of these results, and in so doing, clarify the relationship between the two types of conditions needed.
\end{abstract}

1. Introduction. Let $R^{n}$ denote usual Euclidean $n$-space, and let $F$ be a $R^{n}$-valued function, $F:\left(t_{0}, t_{1}\right) \times R^{n} \rightarrow R^{n}$. Consider the initial value problem

$$
\begin{aligned}
\text { (i) } x^{\prime}(t) & =F(t, x(t)), \quad t \in\left(t_{0}, t_{1}\right), \\
\text { (ii) } x\left(t_{0}\right) & =x_{0} \in R^{n} .
\end{aligned}
$$

A function $x:\left[t_{0}, t_{1}\right) \rightarrow R^{n}$ is a solution of $(*)_{n}$ if $x$ is continuous on $\left[t_{0}, t_{1}\right)$, $x^{\prime}$ exists everywhere on $\left(t_{0}, t_{1}\right)$, and (i) and (ii) are satisfied.

In this note, we clarify the relationship between the modulus of continuity and growth conditions imposed on $F$ which yield Nagumo type uniqueness results for solutions of $(*)_{n}$. In particular, as applications, we indicate generalizations of recent results of Bownds and Metcalf [2], and Rogers [9], both of which extend the classical Nagumo uniqueness theorem.

2. The Nagumo theorem. Nagumo's original result states that if $F$ is continuous on $\left(t_{0}, t_{1}\right) \times R$ and satisfies

$$
|F(t, x)-F(t, y)| \leqslant\left(t-t_{0}\right)^{-1}|x-y|
$$

for $t \in\left(t_{0}, t_{1}\right), x, y \in R$, then $(*)_{1}$ has at most one solution. It is well known that (1) cannot be replaced by

$$
|F(t, x)-F(t, y)| \leqslant(1+\varepsilon)\left(t-t_{0}\right)^{-1}|x-y|
$$

if $\varepsilon>0$. An extension was obtained by Walter and Diaz [4], who proved uniqueness by retaining (1) and replacing the assumption of continuity of $F$ with just the statement that

Presented to the Society, January 27, 1977; received by the editors December 13, 1976 and, in revised form, July 15, 1977.

AMS (MOS) subject classifications (1970). Primary 34A10; Secondary 34A40. 


$$
\operatorname{limit}_{(t, x) \rightarrow\left(t_{0}^{+}, x_{0}\right)} F(t, x) \text { exists; }
$$

the necessity of (3) has been demonstrated by Diaz [3]. Bownds and Metcalf's [2] result (see Example 1 below) allows assumption (2) at the expense of requiring that the limit (3) be approached at a certain minimal rate. Rogers' [9] result (see Example 2) meanwhile assumes that (1) is replaced by the weaker condition

$$
|F(t, x)-F(t, y)| \leqslant\left(t-t_{0}\right)^{-2}|x-y|, \quad 0<t-t_{0} \leqslant 1,
$$

while requiring a more restrictive minimal rate of approach toward some limit for $F$ at $\left(t_{0}, x_{0}\right)$. The theorem given below clarifies the relationship between the modulus of continuity and minimal rate of approach conditions while extending both of these Nagumo type uniqueness theorems.

\section{The main result.}

THEOREM. Let $\varphi$ be a continuous function on $\left[t_{0}, t_{1}\right)$, and differentiable on $\left(t_{0}, t_{1}\right)$ such that $\varphi\left(t_{0}\right)=0, \varphi(t)>0$ for $t>t_{0}$. Suppose further that

(i) $(F(t, x)-F(t, y)) \cdot(x-y) \leqslant \frac{\varphi^{\prime}(t)}{\varphi(t)}\|x-y\|^{2}$,

$$
t \in\left(t_{0}, t_{1}\right), \quad x, y \in R^{n}
$$

and

(ii) there is an $L \in R^{n}$ such that

$$
F(t, x)=L \varphi^{\prime}(t)+o\left(\varphi^{\prime}(t)\right) \text { as } t \rightarrow t_{0}^{+}, x \rightarrow x_{0} .
$$

Then (*) $)_{n}$ has at most one solution.

Here, · and || $\|$ are the usual Euclidean n-space inner product and norm, respectively.

The basic idea employed in the proof is to obtain uniqueness via the classical differential inequality technique, which appears in several places in the literature, for example [1], [6], [8] and [11]. In particular, in [1], Bernfeld, Driver, and Lakshmikantham obtain Bownds and Metcalf's result and Rogers' result as special cases of their general Lyapunov like functioncomparison principle theorem which extends the classical result of Kamke. The distinctive feature of the Bernfeld, Driver, and Lakshmikantham theorem is the introduction of an auxiliary function $B(t)$ such that: for solutions $x(t)$ and $y(t)$, the Lyapunov like function $V(t, x(t), y(t))$ is $o(B(t))$ as $t \rightarrow t_{0}^{+}$; and the only solution of the comparison equation $u^{\prime}=w(t, u)$ which is $o(B(t))$ as $t \rightarrow t_{0}^{+}$is $u \equiv 0$. (We recall that the main hypothesis in such theorems is $D^{+} V(t, x(t), y(t)) \leqslant w(t, V(t, x(t), y(t)))$.) Under these conditions, they carry out a standard differential inequality argument, obtaining uniqueness. In our case, we are able to simplify the proof by essentially incorporating the role of $B(t)$ in the construction of the Lyapunov like function in such a way that we can use the trivial function $w \equiv 0$ in the comparison equation. Thus our result is derived from a possibly less general, but more elementary, 
theorem than that of Bernfeld, Driver, and Lakshmikantham.

Proof OF THE TheOREM. Let $x(t)$ and $y(t)$ be solutions of $(*)_{n}$. Define

$$
v(t)= \begin{cases}\frac{1}{2}[\|x(t)-y(t)\| / \varphi(t)]^{2}, & t \in\left(t_{0}, t_{1}\right), \\ 0, & t=t_{0} .\end{cases}
$$

Writing $x=\left\{x_{i}\right\}, y=\left\{y_{i}\right\}$, and $F=\left\{F_{i}\right\}$, we first note that

$$
\operatorname{limit}_{t \rightarrow t_{0}^{+}} \frac{x_{i}(t)-y_{i}(t)}{\varphi(t)}=\operatorname{limit}_{t \rightarrow t_{0}^{+}} \frac{F_{i}(t, x(t))-F_{i}(t, y(t))}{\varphi^{\prime}(t)}=0,
$$

making use of L'Hospital's rule and hypothesis (ii). Hence $v$ is continuous on $\left[t_{0}, t_{1}\right)$. Now, for $t>t_{0}$,

$$
\begin{aligned}
v^{\prime}(t)= & -\frac{\|x(t)-y(t)\|^{2}}{(\varphi(t))^{3}} \varphi^{\prime}(t) \\
& +\sum_{i=1}^{n} \frac{\left(x_{i}(t)-y_{i}(t)\right)\left(F_{i}(t, x(t))-F_{i}(t, y(t))\right)}{(\varphi(t))^{2}} \leqslant 0,
\end{aligned}
$$

by hypothesis (i). Thus, $v(t)$ is continuous and nonincreasing on $\left[t_{0}, t_{1}\right)$. Since $v$ is nonnegative, $v \equiv 0$ on $\left[t_{0}, t_{1}\right)$, and so

$$
x(t)=y(t) \text { on }\left(t_{0}, t_{1}\right) .
$$

This completes the proof.

4. Some examples. We now show how the results of Bownds and Metcalf [2] and Rogers [9] can be obtained as special cases of our result.

Bownds and Metcalf's [2] result is the $n=1$ case of the following example.

EXAMPLE 1. Let $\alpha>1$, and assume

(i) $(F(t, x)-F(t, y)) \cdot(x-y) \leqslant \frac{\alpha}{t-t_{0}}\|x-y\|^{2}$,

$$
t \in\left(t_{0}, t_{1}\right), \quad x, y \in R^{n},
$$

(ii) $\operatorname{limit}_{(t, x) \rightarrow\left(t_{0}^{+}, x_{0}\right)} F(t, x)\left(t-t_{0}\right)^{1-\alpha}$ exists.

The fact that (*) ${ }_{n}$ has at most one solution follows by using $\varphi(t)=\left(t-t_{0}\right)^{\alpha}$. Rogers' [9] result is the $n=1, \beta=2$, two-sided case of the following example.

EXAMPLE 2. Let $\beta>1$, and assume

(i) $(F(t, x)-F(t, y)) \cdot(x-y) \leqslant \frac{\beta-1}{\left(t-t_{0}\right)^{\beta}}\|x-y\|^{2}$,

$$
0<t-t_{0} \leqslant 1, \quad x, y \in R^{n},
$$

(ii) $\operatorname{limit}_{(t, x) \rightarrow\left(t_{0}^{+}, x_{0}\right)} F(t, x)\left(t-t_{0}\right)^{\beta} \exp \left\{\left(t-t_{0}\right)^{1-\beta}\right\}$ exists.

Choosing $\varphi(t)=(\beta-1)^{-1} \exp \left\{-\left(t-t_{0}\right)^{1-\beta}\right\}$, we see that $(*)_{n}$ has at most one solution.

The following example illustrates a situation in which our theorem predicts 
the uniqueness, but neither the Bownds and Metcalf, nor the Rogers result, is applicable.

EXAMPLE 3. Consider the scalar initial value problem

$$
x^{\prime}(t)=(-\ln t) x(t), \quad t \in(0, T), \quad x(0)=x_{0},
$$

where $T$ is a positive number less than 1 , to be more specifically determined below.

The unique solution of (1), (2) is

$$
x(t)=x_{0} \exp \{t(1-\ln t)\} .
$$

Now, since neither $[(-\ln t) x] t^{1-\alpha}$ (for any $\left.\alpha>1\right),[(-\ln t) x] t^{2} \exp \left\{t^{-1}\right\}$ have a finite limit as $t \rightarrow 0^{+}$, the results of Bownds and Metcalf and Rogers could not be applied here.

To show that our result does predict uniqueness, we begin by observing $t(\ln t)^{2} \rightarrow 0$ as $t \rightarrow 0^{+}$. We choose $T<1$, so that $t(\ln t)^{2} \leqslant 1, t<T$. Let $\varphi(t)=-(\ln t)^{-1}, \varphi(0)=0$. Then $\varphi^{\prime}(t)=1 / t(\ln t)^{2}$, and $\varphi^{\prime}(t) / \varphi(t)=$ $-(t \ln t)^{-1}$. Now since $t(\ln t)^{2} \leqslant 1, t<T$,

$$
\begin{aligned}
F(t, x)-F(t, y)=(-\ln t)(x-y) \leqslant \frac{-1}{t \ln t}( & x-y), \\
0 & <t<T, \text { and } x>y .
\end{aligned}
$$

Further,

$$
\frac{F(t, x)}{\varphi^{\prime}(t)}=\frac{(-\ln t) x}{1 / t(\ln t)^{2}}=-t(\ln t)^{3} x \rightarrow 0 \quad \text { as } t \rightarrow 0^{+} .
$$

Thus (i) and (ii) are verified, and so the Theorem can be applied.

Now let $K>0$. Using $\varphi(t)=(-1 / \ln t)^{K}$, similarly to the above example, yields the following uniqueness criterion: Suppose $F:(0, T) \times R^{n} \rightarrow R^{n}$ satisfies

(i) $(F(t, x)-F(t, y)) \cdot(x-y) \leqslant-K \ln t\|x-y\|^{2}, t \in(0, T), x, y \in$ $R^{n}$,

(ii) $t(\ln t)^{K+1} F(t, x) \rightarrow L$ as $t \rightarrow 0^{+}, x \rightarrow x_{0}$, for some $L \in R^{n}$.

Then the initial value problem $x^{\prime}(t)=F(t, x(t)), x(0)=x_{0}$ has at most one solution.

Finally, in addition to the example of Diaz noted earlier, we mention the following simple example indicating the necessity of the conditions in the Theorem.

Let $\varphi(t)$ satisfy the conditions of the Theorem. Consider the intitial value problem

$$
x^{\prime}(t)=\frac{\varphi^{\prime}(t)}{\varphi(t)} x(t), \quad t>0, \quad x(0)=0
$$

Then (i) is satisfied, but uniqueness does not hold as the functions $K \varphi(t), K$ constant, are solutions. Now, (ii) does not hold for $\varphi(t)$, but is valid for $\ln \varphi(t)$, for example; this gives some idea of the sharpness of condition (ii). 
5. Comparison with other known uniqueness results. In [5], Hille gives a two-sided existence and uniqueness theorem in which the assumptions are: in some domain $\left|t-t_{0}\right| \leqslant a,\left\|x-x_{0}\right\| \leqslant b$ :

(1) $\|F(t, x)-F(t, y)\| \leqslant w\left(\left|t-t_{0}\right|,\|x-y\|\right), t \neq t_{0}$,

(2) $F(t, x)$ is continuous,

where $w$ is an auxiliary function. As such $w$ must satisfy a number of conditions, two of which are:

(i) There exists a constant $c>0$ such that $w(\tau, c \tau) \leqslant c, 0<\tau \leqslant a$.

(ii) For any nonnegative continuous function $h$ on $[0, a]$ with $h(0)=0$ and $h(\tau) / \tau \rightarrow 0$ as $\tau \rightarrow 0, w(\tau, h(\tau)) \rightarrow 0$ as $\tau \rightarrow 0$.

Now in the two-sided version of our Theorem this function would have the form

(iii) $w(\tau, u)=\psi^{\prime}(\tau) u / \psi(\tau)$

(compare (1) above and hypothesis (i) of our Theorem) where $\psi(\tau)=$ $\varphi\left(\tau+t_{0}\right)$. However, under the assumptions in our Theorem, this function would not necessarily satisfy conditions (i) and (ii) above. Indeed, this is the case in our Example 2.

Furthermore, Hille's proof centers on the construction of an increasing sequence $\left\{h_{n}\right\}$ of nonnegative functions satisfying $h_{n}(0)=0$ and which converges to a solution of $u^{\prime}=w(\tau, u)$; the first of these is $h_{0}(\tau)=\| x\left(t_{0}+\tau\right)$ $-y\left(t_{0}+\tau\right) \|$, where $x(t)$ and $y(t)$ are solutions of the initial value problem. Uniqueness follows by invoking the hypothesis that the only such solution of $u^{\prime}=w(\tau, u)$ is identically zero. The argument for convergence rests on the fact that $h_{n}(\tau) / \tau \rightarrow 0$, as $\tau \rightarrow 0^{+}$, all $n$. This is not necessarily true under the assumptions of our Theorem.

Clearly the uniqueness part of Hille's theorem is not a special case of ours, since he allows $w$ functions not necessarily of the form (iii). From this and what we have mentioned above, it appears that neither result contains the other as a special case, although there is some overlap in applicability of the two results.

The uniqueness theorems presented in Walter [10] require only a modulus of continuity condition like (1), with different assumptions on the function $w$. For example, the one-sided one dimensional result requires (for the initial value problem with $\left.t_{0}=0\right) F(t, x)-F(t, y) \leqslant w(t, x-y)$ for $x>y$ and $t \in(0, T]$, where $w$ must satisfy the following condition: for arbitrary $\varepsilon>0$, there is a $\delta>0$ such that the differential inequality $u^{\prime}>w(t, u)$ has a solution on $(0, T]$ satisfying $\delta \leqslant u \leqslant \varepsilon$. That neither this criterion nor a slight extension given subsequently includes the Nagumo condition is noted by Walter as he mentions the fact that the Nagumo function $w(t, u)=u / t$ (by itself) does not yield uniqueness.

In conclusion, neither of the uniqueness theorems given by Hille and Walter take into account the degree of continuity of $F$ at the initial point (in addition to modulus of continuity condition in the second variable) needed to obtain uniqueness results of the Nagumo type. That our theorem does this job is its distinguishing aspect. 
The author wishes to acknowledge Professor T. G. Hallam for helpful discussions in preparing this note.

\section{REFERENCES}

1. S. R. Bernfeld, R. D. Driver and V. Lakshmikantham, Uniqueness for ordinary differential equations, Math. Systems Theory 9 (1976), 359-367.

2. J. M. Bownds and F. T. Metcalf, An extension of the Nagumo uniqueness theorem, Proc. Amer. Math. Soc. 27 (1971), 313-316.

3. J. B. Diaz, On existence, uniqueness, and numerical evaluation of solutions of ordinary and hyperbolic differential equations, Ann. Mat. Pura Appl. 52 (1960), 136-181.

4. J. B. Diaz and W. L. Walter, On uniqueness theorems for ordinary differential equations and for partial differential equations of hyperbolic type, Trans. Amer. Math. Soc. 96 (1960), 90-100.

5. E. Hille, Lectures on ordinary differential equations, Addison-Wesley, Reading, Mass., 1969.

6. V. Lakshmikantham and S. Leela, Differential and integral inequalities, Vol. 1, Academic Press, New York, 1969.

7. M. Nagumo, Eine hinreichende Bedingung für die Unität der Lösung von Differentialgleichungen erster Ordnung, Japan. J. Math. 3 (1926), 107-112.

8. H. Okamura, Condition necéssaire et suffisante remplie par les équations différentielles ordinaires sans points de Peano, Mem. Coll. Sci. Kyoto Imp. Univ. Ser. A 24 (1942), 21-28.

9. T. Rogers, On Nagumo's condition, Canad. Math. Bull. 15 (1972), 606-611.

10. W. Walter, Differential and integral inequalities, Springer-Verlag, New York, 1970.

11. T. Yoshizawa, Stability theory by Liapunov's second method, Math. Soc. Japan, Tokyo, 1966.

Department of Mathematics, University of Georgia, Athens, Georgia 30602 